\title{
Synaptotagmin 10-mediated release
}

Synaptotagmin 1 (SYT1), SYT2, SYT7 and SYT9 act as calcium sensors in fast synaptic and neuroendocrine exocytosis. Mammals express several other SYTs, including SYT10, but the functions of these proteins are unknown. Südhof and colleagues now show that SYT10 regulates a calcium-dependent exocytic pathway that is functionally distinct from that controlled by SYT1.

To examine the biological role of SYT10, the authors knocked out Syt10 in mice. Constitutive Syt10 animals showed impairments in locating hidden food, indicative of a defect in olfaction. This observation

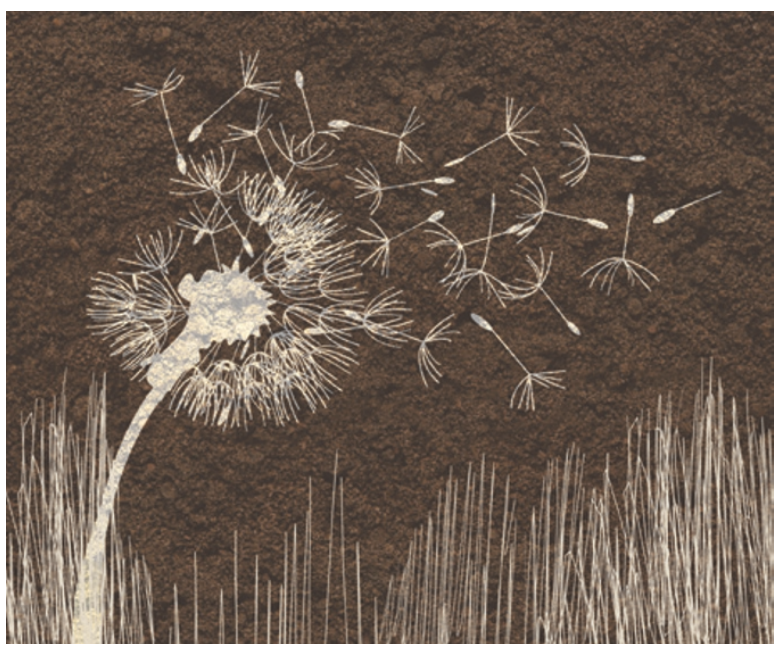

was consistent with the normal expression of SYT10, which is highest in the olfactory bulb.

In electrophysiological experiments, cultured olfactory bulb neurons - comprising mainly excitatory mitral neurons and inhibitory granule cell neurons - from conditional Syt $10^{-/-}$mice showed a decrease in synaptic strength that extended to excitatory and inhibitory synapses. This finding suggested that deficits in synaptic transmission underlie the behavioural phenotype observed in Syt $10^{-/-}$mice. Of note, cultured Syt $1^{-/-}$olfactory bulb neurons showed a notably different electrophysiological phenotype to Syt $10^{-/-}$olfactory bulb neurons.

Capacitance measurements from slice recordings and immunostaining data indicated that Syt $10^{-/-}$olfactory bulb neurons were smaller than those from wild-type mice. Moreover, Syt $10^{-1-}$ mitral neurons showed a dramatic reduction in arborization, but normal synaptic density in dendrites. These findings suggest that the synaptic transmission deficits were attributable to a decreased size and arborization of olfactory bulb neurons, rather than a specific effect on synapses.

The authors investigated how the SYT10 deficiency mediated these effects. They found that olfactory bulb neurons secreted insulin-like growth factor 1 (IGF1) following mild depolarization, and that this release was impaired in Syt10 $0^{-/-}$olfactory bulb neurons. Furthermore, tetrodotoxin-silenced olfactory bulb neurons exhibited a similar electrophysiological phenotype to Syt $10^{-/-}$olfactory bulb neurons and also had impaired IGF1 secretion. Thus, the absence of SYT10 seems to exert its effects through a reduction of IGF1 signalling.

In mitral neurons, SYT10 and IGF1 colocalized in vesicles - in cell bodies and dendrites - that did not express various established synaptic vesicle-related proteins. Moreover, depolarization of these cells caused rapid exoctyosis of IGF1 in a calcium-dependent manner.

This study shows that SYT10 regulates a previously unidentified pathway of calcium-dependent exocytosis. Strikingly, this pathway occurs outside the synapse and constitutes the first known regulatory mechanism for IGF1 secretion.

Darran Yates

ORIGINAL RESEARCH PAPER Cao, P., Maximov, A. \& Südhof, T. C. Activity-dependent IGF-1 exocytosis is controlled by the $\mathrm{Ca}^{2+}$-sensor synaptotagmin-10. Cell 145, 300-311 (2011) 